\title{
Pacific
}

Journal of

Mathematics

\section{A FROBENIUS PROBLEM ON THE KNOT SPACE}

\author{
RON G. WANG
}




\title{
A FROBENIUS PROBLEM ON THE KNOT SPACE
}

\author{
R.G. WANG
}

\begin{abstract}
According to J.-L. Brylinski, there is a natural almost complex structure $J$ on the space $K$ of all knots in the Euclidean space $R^{3}$. The almost complex structure is formally integrable on $K$, i.e, the Nijenhuis tensor of $J$ vanishes. The problem is whether $J$ is integrable and hence $K$ is a complex manifold. In this paper, we study the integrability of $J$ explicitly in view point of a Frobenius problem.
\end{abstract}

\section{Introduction}

A knot is by definition a smooth imbedded circle in the Euclidean space $R^{3}$. The knot space is the space of all knots. In this paper, we study an integrability problem on the knot space which is as follows: According to Brylinski $[\mathbf{3}, \mathbf{4}]$, for any $\gamma \in K$, the tangent space $T_{\gamma} K$ is the space of sections of the normal bundle of $\gamma$ in $R^{3}$. A natural almost complex structure $J$ is defined on $K$ as a rotation of $\frac{\pi}{2}$ in the normal plane bundle. $J$ is formally integrable on $K$, i.e, the Nijenhuis tensor of $J$ vanishes. Compared to the well-known theorem of Newlander-Nirenberg [17], the problem is whether $J$ is integrable and hence $K$ is a complex manifold.

A result of Drinfeld and LeBrun $[\mathbf{3}, 4]$ is that $J$ is weakly integrable on the space $K_{0}$ of real analytic knots, i.e., there are enough holomorphic functions on each local chart of $K_{0}$. In Lempert [15], the theory of twistor CR-manifolds is used to prove that $J$ is weakly integrable on the space of real analytic knots in a real analytic 3 -manifold with a real analytic metric. It is also proved that $J$ is not integrable on the space $K$ and $K_{0}$, i.e., there is no open set $U \neq \phi$ on the knot space which is biholomorphic to an open set in $T_{\gamma} K$ or $T_{\gamma} K_{0}$. LeBrun [14] has a similar result on the so-called space of world-sheets which are time-like 2-surfaces in 4-manifold with a Lorentzian metric.

In this paper, we define a natural local coordinate system on $K$ and study the integrability of $J$ explicitly in view point of a Frobenius problem. It will be shown that in the local coordinate system $J$ can be written explicitly to see that it is real analytic and the $\bar{\partial}$-equation can be complexified to obtain a Frobenius problem and the Frobenius problem can be further reduced to a first order nonlinear partial differential equation in two dimensions. In the 
case $K_{0}$, the equation is solvable and hence $J$ is weakly integrable by the theorem of Cauchy-Kowalewska. In the case $K$, the equation is not solvable and thus the Frobenius problem is not integrable. (This does not implies that $J$ is not integrable.) It is also explained that why the holomorphic functions on $K_{0}$ fail to make a local chart by the implicit function theorem. Acknowledgement. The author wishes to thank Professor C.H. Taubes for introducing him to the problem and helpful advices and Professor J.-L. Brylinski for valuable conversations and encouragement, and also Professor L. Lempert and J. Bernstein for discussions.

\section{The Knot Space $K$}

In this section, some basic properties on the knot space $K$ are collected and a natural coordinate system on $K$ is defined on $K$. To formulate the almost complex structure $J$ on $K$, the local basis on each of the local chart is also explicitly given. For a general knowledge on the knot space $K$, the reader may refer to Brylinski [5], which serves as the background of the paper.

2.1. The knot space $K$. The knot space $K$ is roughly speaking the space of all knots in $R^{3}$. A precise identification of the space $K$ is given as follows.

The knot space $K$ has a close relation with the loop space $L$, i.e., the space of all smooth maps from the standard circle $S^{1}$ to $R^{3}$, with the topology of uniform convergence of the map and all its derivatives. It is well-known that $L$ is a Fréchet space, and the orientation preserving diffeomorphism group of $S^{1}$ acts on $L$ as a reparametrization. Restricted on the space $L^{*}$ of imbedded loops, the action is free and the quotient space is a smooth Fréchet manifold. The knot space $K$ is thus defined to be the quotient space.

An element in $K$ is a closed oriented imbedded curve in $R^{3}$. For any $\gamma \in K$, denote $l$ the arc length of $\gamma$ and $s$ an arc-length parametrization of $\gamma$. For convenience, a parametrization $\theta$ of $\gamma$ is called standard, if

$$
\frac{d s}{d \theta}=l,(0 \leq \theta \leq 1)
$$

An elementary fact is that different arc-length or standard parametrizations of $\gamma$ differ only by a constant.

For any $\gamma \in K$, let $N_{\gamma}$ denote the normal bundle of $\gamma$ in $R^{3}$. A basic fact is that the tangent space $T_{\gamma} K$ is the space $\Gamma\left(N_{\gamma}\right)$ of sections of $N_{\gamma}$. This can be understood as follows: Since $L^{*}$ is an open submanifold in $L$, for any $\gamma \in L^{*}$

$$
T_{\gamma} L^{*} \simeq C^{\infty}\left(S^{1}, R^{3}\right)
$$

Modulo the tangent factor to the knot, $T_{\gamma} K=\Gamma\left(N_{\gamma}\right)$. 
For any $\gamma \in K$, denote by $N_{\delta}(\gamma)$ the tubular neighborhood of $\gamma$ with radius $\delta$ in $R^{3}$. Note that, when $\delta>0$ is small, $N_{\delta}(\gamma)$ is imbedded in $R^{3}$, the space $\mathcal{N}_{\delta}(\gamma)$ of knots in $R^{3}$ with image in $N_{\delta}(\gamma)$ is an open neighborhood of $\gamma$ in $K$. Note also that $\mathcal{N}_{\delta}(\gamma)$ can be identified as the space of sections $h$ of $N_{\gamma}$ with $C^{0}$-norm $\|h\|_{C^{0}}<\delta$.

$$
\mathcal{N}_{\delta}(\gamma) \simeq\left\{h \in \Gamma\left(N_{\gamma}\right):\|h\|_{C^{0}}<\delta\right\} .
$$

Similarly, the space $\mathcal{N}_{\delta}^{1}(\gamma)$ of knots in $R^{3}$, which can be identified as

$$
\mathcal{N}_{\delta}^{1}(\gamma) \simeq\left\{h \in \Gamma\left(N_{\gamma}\right):\|h\|_{C^{1}}<\delta\right\}
$$

is also an open neighborhood of $\gamma$ in $K$.

2.2. A local coordinate system on $K$. To define a local coordinate system on the knot space $K$, recall the basic theory of frénet of curves in $R^{3}$ as follows. Note that an element $\gamma \in K$ is a closed imbedded curve in $R^{3}$, the curvature $\kappa$ of $\gamma$ is a well-defined continuous function along $\gamma$. $\kappa$ has nonnegative values and may be zero somewhere on $\gamma$. Denote by $K^{*}$ the space of knots in $R^{3}$ with curvature $\kappa>0$ everywhere, i.e,

$$
K^{*}=\{\gamma \in K: \kappa>0\} .
$$

There is first the following:

Lemma 2.1. The space $K^{*}$ is open and dense in $K$.

Proof. Clearly $K^{*}$ is an open set in $K$. To show that $K^{*}$ is dense in $K$, the idea is that, for any $\gamma \in K$, even $\kappa$ vanishes somewhere on $\gamma$, a generic small twist of the curve has positive curvature everywhere. In another word, a certain generic perturbation of $\gamma$ is in $K^{*}$.

To describe the perturbation, note first that $N_{\gamma}$ is a trivial plane bundle, there are two sections $\tilde{e}_{2}, \tilde{e}_{3}$ of $N_{\gamma}$ which form a basis of $\Gamma\left(N_{\gamma}\right)$. Let $\theta$ be a standard parametrization of $\gamma$; then the perturbation $\tilde{\gamma}$ is a twist by the normal frame field as follows:

$$
\tilde{\gamma}(\theta)=\gamma(\theta)+f_{2}(\theta) \tilde{e}_{2}(\theta)+f_{3}(\theta) \tilde{e}_{3}(\theta),
$$

where $f_{2}, f_{3}$ are smooth periodic functions in $\theta$. Note that $\frac{d \tilde{\gamma}}{d \theta}$ involves $f_{2}, f_{3}$ and their first derivatives, when $\delta>0$ is small, and

$$
\left\|f_{2}\right\|_{C^{1}}<\delta,\left\|f_{3}\right\|_{C^{1}}<\delta
$$

$\frac{d \tilde{\gamma}}{d \theta} \neq 0$ everywhere. Denote by $\tilde{s}$ an arc-length parametrization of $\tilde{\gamma}$. Then for a generic perturbation $\left(f_{2}, f_{3}\right), \frac{d^{2} \tilde{\gamma}}{d \tilde{s}^{2}} \neq 0$ everywhere. Thus $\kappa(\tilde{\gamma})>0$, $\tilde{\gamma} \in K^{*}$. This shows that $K^{*}$ is dense in $K$. Lemma 2.1 is proved. 
To define a local coordinate system on $K$, for any $\gamma \in K^{*}$, fix an arclength parametrization $s$ and a standard parametrization $\theta$ of $\gamma$. Note that the Frenét frame $\left\{e_{1}, e_{2}, e_{3}\right\}$ is well-defined along $\gamma$, where

$$
\left\{\begin{array}{l}
e_{1}=\frac{d \gamma}{d s} \\
\frac{d e_{1}}{d s}=\kappa e_{2} \\
e_{3}=e_{1} \times e_{2} .
\end{array}\right.
$$

Recall the following Frenét formula:

$$
\left\{\begin{array}{l}
\frac{d e_{1}}{d s}=\kappa e_{2} \\
\frac{d e_{2}}{d s}=-\kappa e_{1}+\tau e_{3} \\
\frac{d e_{3}}{d s}=-\tau e_{2}
\end{array}\right.
$$

Recall that the open neighborhood $\mathcal{N}_{\delta}(\gamma)$ of $\gamma$ is identified as (2.1). For any $\tilde{\gamma} \in \mathcal{N}_{\delta}(\gamma), \tilde{\gamma}$ correspondences to a section $z(\theta) \in \Gamma\left(N_{\gamma}\right)$. Note that $z(\theta)$ can be written as

$$
z(\theta)=x(\theta) e_{2}+y(\theta) e_{3}
$$

where $x(\theta), y(\theta)$ are smooth periodic functions in $\theta$. Expand $x(\theta)$ and $y(\theta)$ as Fourier series

$$
\begin{aligned}
& x(\theta)=x_{0}+\sum_{k=1}^{\infty} x_{2 k-1} \sin (2 k \pi \theta)+x_{2 k} \cos (2 k \pi \theta), \\
& y(\theta)=y_{0}+\sum_{k=1}^{\infty} y_{2 k-1} \sin (2 k \pi \theta)+y_{2 k} \cos (2 k \pi \theta),
\end{aligned}
$$

then a local coordinate of $\tilde{\gamma}=\gamma+z(\theta) \in \mathcal{N}_{\delta}(\gamma)$ can be given as the Fourier coefficients $\left\{x_{k}, y_{k}: k \in N\right\}$.

To define the local coordinate system on $K$, it is left to show that the collection

$$
\left\{\mathcal{N}_{\delta}(\gamma): \gamma \in K^{*}, \delta>0\right\}
$$

is an open cover on $K$. Needless to say, in (2.6), $\delta>0$ is chosen small so that the tubular neighborhood $N_{\delta}(\gamma)$ is imbedded in $R^{3}$. 
Lemma 2.2. $K=\cup_{\gamma \in K^{*}, \delta>0} \mathcal{N}_{\delta}(\gamma)$.

Proof. For any $\gamma \in K$, choose $\delta>0$ and a sequence $\left\{\gamma_{n}\right\}$ in $K^{*}$ so that $N_{\delta}(\gamma)$ is imbedded and $\gamma_{n} \rightarrow \gamma$ in $C^{0}$-norm. Choose $n$ large such that $N_{\frac{\delta}{2}}\left(\gamma_{n}\right)$ is also imbedded; then $\gamma \in \mathcal{N}_{\frac{\delta}{2}}\left(\gamma_{n}\right)$.

Similarly, the collection

$$
\left\{\mathcal{N}_{\delta}^{1}(\gamma): \gamma \in K^{*}, \delta>0\right\}
$$

is an open cover on $K$. Thus (2.7) also defines a local coordinate system on $K$. This is the local coordinate system we will use.

2.3. A local basis on the local patch. To formulate the almost complex structure $J$ in local coordinates, a local basis $\left\{X_{k}, Y_{k}: k \in N\right\}$ on $K$ will be defined in this section. It will be also shown that $\left\{X_{k}, Y_{k}: k \in N\right\}$ is the local basis, i.e., $X_{k}=\partial_{x_{k}}, Y_{k}=\partial_{y_{k}}$ for all $k \in N$.

To define $X_{0}$, consider the normal vector field $e_{2}=e_{2}(\theta)$ along $\gamma$. Note that $e_{2}$ can be regarded as a tangent vector on $K$ at $\gamma$. It is defined that $X_{0}(\gamma)=e_{2}$. For any $\tilde{\gamma} \in \mathcal{N}_{\delta}^{1}(\gamma)$, to define $X_{0}(\tilde{\gamma})$, translate the vector field $e_{2}=e_{2}(\theta)$ along $\gamma$ onto $\tilde{\gamma}$. Note that $e_{2}$ may not remain in $T_{\tilde{\gamma}} K$, i.e., $e_{2}(\theta)$ may have both normal component $\bar{e}_{2}$ and tangential component $e_{2}^{T}$ along $\tilde{\gamma}$. It is defined that $X_{0}=\bar{e}_{2} . \bar{e}_{2}$ will be explicitly computed later.

To define $Y_{0}$ on $\mathcal{N}_{\delta}^{1}(\gamma)$, consider the normal vector field $e_{3}=e_{3}(\theta)$ along $\gamma$. The translated vector field $e_{3}(\theta)$ along $\tilde{\gamma}$ may have both normal component $\bar{e}_{3}$ and tangential component $e_{3}^{T}$. It is defined that $Y_{0}=\bar{e}_{3} . \bar{e}_{3}$ will be also explicitly computed later.

Similarly, for any $k \in N$, consider the translated vector field $\sin (2 k \pi \theta) e_{2}(\theta)$ along $\tilde{\gamma}$. Note that the normal component is $\sin (2 k \pi \theta) \bar{e}_{2}$ and the tangential component is $\cos (2 k \pi \theta) e_{2}^{T}$. It is defined that

$$
X_{2 k-1}=\sin (2 k \pi \theta) \bar{e}_{2} .
$$

There are also the following definitions:

$$
\begin{gathered}
X_{2 k}=\cos (2 k \pi \theta) \bar{e}_{2}, Y_{2 k-1}=\sin (2 k \pi \theta) \bar{e}_{3}, \\
Y_{2 k}=\cos (2 k \pi \theta) \bar{e}_{3}(k \in N) .
\end{gathered}
$$

Proposition 2.3. $\left\{X_{k}, Y_{k}: k \in N\right\}$ defined above is the local basis on the local patch $\mathcal{N}_{\delta}^{1}(\gamma)$ when $\delta>0$ is small, i.e.,

$$
X_{k}=\partial_{x_{k}}, Y_{k}=\partial_{y_{k}}
$$


where $\left\{x_{k}, y_{k}\right\}$ is the local coordinates defined as (2.5).

Proof. Notice that $X_{k}, Y_{k}$ are in fact inherited from the base vectors on the loop space $L$. To be precise, let

$$
L^{\prime}=\left\{\gamma \in L^{*}: \kappa(\gamma)>0\right\} .
$$

Then $L^{\prime}$ is an open subset in $L$. For any $\gamma \in L^{\prime}$, let $\left\{e_{1}, e_{2}, e_{3}\right\}$ be the Frenét frame along $\gamma$. Note that, for any $\tilde{\gamma}$ in a neighborhood of $\gamma$ in $L, \tilde{\gamma}$ can be written as

$$
\tilde{\gamma}=\gamma+\sum_{i=1}^{3} h_{i} e_{i}
$$

for some smooth periodic functions $h_{1}, h_{2}, h_{3}$. Thus, a local coordinate of $\tilde{\gamma}$ can be given as the coefficients of the Fourier expansion of $h=\left(h_{1}, h_{2}, h_{3}\right)$; $e_{1}, e_{2}, e_{3}$ are all local base vectors on $L$. Modulo the factor with values in the Virasoro algebra, $\bar{e}_{2}, \bar{e}_{3}$ are both local base vectors on $\mathcal{N}_{\delta}^{1}(\gamma)$,

$$
\bar{e}_{2}=\partial_{x_{0}}, \bar{e}_{3}=\partial_{y_{0}}
$$

Similarly, the other $X_{k}, Y_{k}$ 's are also base vectors on $\mathcal{N}_{\delta}^{1}(\gamma)$,

$$
X_{k}=\partial_{x_{k}}, Y_{k}=\partial_{y_{k}}(k \in N) .
$$

Remark. Notice that $\left\|e_{2}^{T}\right\|_{C^{0}}$ and $\left\|e_{3}^{T}\right\|_{C^{0}}$ involve the first derivatives of $x(\theta)$ and $y(\theta)$. To ensure $\bar{e}_{2}, \bar{e}_{3} \neq 0$ and linear independent along $\tilde{\gamma}, \bar{e}_{2}$ and $\bar{e}_{3}$ are defined only on the small local patch $\mathcal{N}_{\delta}^{1}(\gamma)$. On the other hand, it is a remark that these local patchs do give an open cover on $K$ and thus defines a local coordinate system on $K$. The proof is similar to that of Lemma 2.2.

$\bar{e}_{2}$ and $\bar{e}_{3}$ are now explicitly computed as follows. For $\gamma \in K^{*}$, denote by $l, \kappa, \tau$ the arc length, curvature and torsion of $\gamma$, and $s, \theta$ an arc-length and standard parameter of $\gamma$, also $\left\{e_{1}, e_{2}, e_{3}\right\}$ the Frenét frame along $\gamma$. For any $\tilde{\gamma} \in \mathcal{N}_{\delta}^{1}(\gamma)$

$$
\tilde{\gamma}=\gamma+x(\theta) e_{2}+y(\theta) e_{3}
$$

let $\tilde{s}$ denote the arc-length parametrization of $\tilde{\gamma}, \tilde{e}_{1}=\frac{d \tilde{\gamma}}{d \tilde{s}}$ the unit tangent field along $\tilde{\gamma}$.

To compute $\bar{e}_{2}$ and $\bar{e}_{3}$, differentiate (2.11). By the Frenét formula,

$$
\tilde{e}_{1} \frac{d \tilde{s}}{d \theta}=l(1-\kappa x) e_{1}+\left(x^{\prime}-l \tau y\right) e_{2}+\left(y^{\prime}+l \tau x\right) e_{3}
$$

For convenience, introduce

$$
\lambda_{1}=l(1-\kappa x), \lambda_{2}=x^{\prime}-l \tau y, \lambda_{3}=y^{\prime}+l \tau x
$$


then there are the following identities:

$$
\begin{gathered}
\frac{d \tilde{s}}{d \theta}=\left(\lambda_{1}^{2}+\lambda_{2}^{2}+\lambda_{3}^{2}\right)^{\frac{1}{2}} \\
\tilde{e}_{1}=\left(\lambda_{1} e_{1}+\lambda_{2} e_{2}+\lambda_{3} e_{3}\right) /\left(\lambda_{1}^{2}+\lambda_{2}^{2}+\lambda_{3}^{2}\right)^{\frac{1}{2}} .
\end{gathered}
$$

Notice that

$$
\begin{aligned}
& \bar{e}_{2}=e_{2}-\left\langle e_{2}, \tilde{e}_{1}\right\rangle \tilde{e}_{1}, \\
& \bar{e}_{3}=e_{3}-\left\langle e_{3}, \tilde{e}_{1}\right\rangle \tilde{e}_{1},
\end{aligned}
$$

$\bar{e}_{2}$ and $\bar{e}_{3}$ are given as:

$$
\begin{gathered}
\bar{e}_{2}=\left[-\lambda_{1} \lambda_{2} e_{1}+\left(\lambda_{1}^{2}+\lambda_{3}^{2}\right) e_{2}-\lambda_{2} \lambda_{2} e_{3}\right] /\left(\lambda_{1}^{2}+\lambda_{2}^{2}+\lambda_{3}^{2}\right) \\
\bar{e}_{3}=\left[-\lambda_{1} \lambda_{3} e_{1}-\lambda_{2} \lambda_{3} e_{2}+\left(\lambda_{1}^{2}+\lambda_{2}^{2}\right) e_{3}\right] /\left(\lambda_{1}^{2}+\lambda_{2}^{2}+\lambda_{3}^{2}\right) .
\end{gathered}
$$

Notice that both $\bar{e}_{2}$ and $\bar{e}_{3}$ are linear combinations of $e_{1}, e_{2}, e_{3}$ with coefficients which are real analytic functions in $\kappa, \tau, x(\theta), y(\theta)$ and the first derivatives $x^{\prime}(\theta), y^{\prime}(\theta)$.

\section{The almost complex structure $\mathbf{J}$}

On the knot space $K$, there is a genuine almost complex structure $J$. Recall that, for any $\gamma \in K, T_{\gamma} K=\Gamma\left(N_{\gamma}\right) . J_{\gamma}$ is defined as the rotation of $\frac{\pi}{2}$ in the plane bundle. In [3-5], it is proved by Brylinski that $J$ is formally integrable, i.e., the Nijenhuis tensor of $J$ vanishes on $K$. In this section, $J$ is formulated explicitly in local coordinates. This means to compute the action of $J$ on the local basis $\left\{X_{k}, Y_{k}\right\}$ defined as (2.8) and (2.9). In this way $J$ is shown real analytic on $K$.

To compute $J\left(X_{k}\right)$ and $J\left(Y_{k}\right)$, for any $\gamma \in K^{*}$, fix a standard parametrization $\theta$ for $\gamma$ and the Frenét frame $\left\{e_{1}, e_{2}, e_{3}\right\}$ along $\gamma$. For any $\tilde{\gamma} \in \mathcal{N}_{\delta}^{1}(\gamma)$,

$$
\tilde{\gamma}=\gamma+x(\theta) e_{2}+y(\theta) e_{3}
$$

let $\tilde{s}$ be the arc-length parametrization for $\tilde{\gamma}$ and $\tilde{e}_{1}=\frac{d \tilde{\gamma}}{d \tilde{s}}$.

Recall that $\tilde{e}_{1}, \bar{e}_{2}$ and $\bar{e}_{3}$ are computed as (2.13) and (2.14). Since $J$ is the rotation of $\frac{\pi}{2}$,

$$
J\left(\bar{e}_{2}\right)=\tilde{e}_{1} \times \bar{e}_{2}
$$

$$
J\left(\bar{e}_{3}\right)=\tilde{e}_{1} \times \bar{e}_{3}
$$


Substituting (2.13) and (2.14) to (3.1), $J\left(\bar{e}_{2}\right)$ and $J\left(\bar{e}_{3}\right)$ are computed as follows:

$$
\begin{aligned}
& J\left(\bar{e}_{2}\right)=\left(\lambda_{2} e_{1}-\lambda_{1} e_{2}\right) /\left(\lambda_{1}^{2}+\lambda_{2}^{2}+\lambda_{2}^{2}\right), \\
& J\left(\bar{e}_{3}\right)=\left(\lambda_{1} e_{3}-\lambda_{3} e_{1}\right) /\left(\lambda_{1}^{2}+\lambda_{2}^{2}+\lambda_{3}^{2}\right),
\end{aligned}
$$

where $\lambda_{1}, \lambda_{2}$ and $\lambda_{3}$ are given as (2.12). Written as linear combinations

$$
J\left(\bar{e}_{2}\right)=a e_{2}+b e_{3}, J\left(\bar{e}_{3}\right)=c \bar{e}_{2}+d \bar{e}_{3},
$$

the coefficients are then given as follows:

$$
\begin{gathered}
a=\frac{\lambda_{2} \lambda_{3}}{\lambda_{1}\left(\lambda_{1}^{2}+\lambda_{2}^{2}+\lambda_{3}^{2}\right)^{\frac{1}{2}}}, \\
b=\frac{\lambda_{1}^{2}+\lambda_{3}^{2}}{\lambda_{1}\left(\lambda_{1}^{2}+\lambda_{2}^{2}+\lambda_{3}^{2}\right)^{\frac{1}{2}}}, \\
c=-\frac{\lambda_{1}^{2}+\lambda_{2}^{2}}{\lambda_{1}\left(\lambda_{1}^{2}+\lambda_{2}^{2}+\lambda_{3}^{2}\right)^{\frac{1}{2}}}, \\
d=-\frac{\lambda_{2} \lambda_{3}}{\lambda_{1}\left(\lambda_{1}^{2}+\lambda_{2}^{2}+\lambda_{3}^{2}\right)^{\frac{1}{2}}}
\end{gathered}
$$

Let $A$ denote the $2 \times 2$ matrix defined as

$$
A=\left(\begin{array}{ll}
a & b \\
c & d
\end{array}\right) .
$$

Note that, for any $X=g_{2} \bar{e}_{2}+g_{3} \bar{e}_{3} \in T_{\gamma} K$,

$$
J X=\tilde{e}_{1} \times X=g_{2} \bar{e}_{2}+g_{3} \bar{e}_{3} .
$$

Denote by $X=\left(g_{2}, g_{3}\right), J$ is then given as

$$
J X=X A \text {. }
$$

$J$ is represented by the matrix $A$ and can be compared to the almost complex structure in two dimensions.

At the origin of $\mathcal{N}_{\delta}^{1}(\gamma), A$ is the standard matrix

$$
A_{\gamma}=\left(\begin{array}{cc}
0 & 1 \\
-1 & 0
\end{array}\right) \text {. }
$$


Note that $A$ is a $2 \times 2$ matrix with entries which are real analytic functions in $\kappa, \tau, x(\theta), y(\theta)$ and the first derivatives $x^{\prime}(\theta), y^{\prime}(\theta) . J$ is a well-defined and smooth almost complex structure on $K$. There is further the following:

Proposition 3. $J$ is a real analytic almost complex structure on $K$.

Proof. With $J$ given explicitly as above, the proof is omitted.

Remark. Since $A$ involves the first derivatives $x^{\prime}(\theta)$ and $y^{\prime}(\theta)$, for any $\gamma \in K$,

$$
J_{\gamma}: T_{\gamma} K \rightarrow T_{\gamma} K
$$

make sense as an endormorphism only when $K$ is equipped with the smooth Fréchet topology.

To end the section, the formula (3.7) is explained as follows. Note that for fixed $\tilde{\gamma} \in \mathcal{N}_{\delta}^{1}(\gamma)$, the entries of $A$ are smooth periodic functions. Expand the entries as Fourier series

$$
\begin{aligned}
& a=a_{0}+\sum_{k=1}^{\infty} a_{2 k-1} \sin (2 k \pi \theta)+a_{2 k} \cos (2 k \pi \theta), \\
& b=b_{0}+\sum_{k=1}^{\infty} b_{2 k-1} \sin (2 k \pi \theta)+b_{2 k} \cos (2 k \pi \theta),
\end{aligned}
$$

then $J\left(\bar{e}_{2}\right)$ is actually given as

$$
J\left(\bar{e}_{2}\right)=a \bar{e}_{2}+b \bar{e}_{3}=\sum_{k=0}^{\infty} a_{k} X_{k}+b_{k} Y_{k} .
$$

$J\left(\bar{e}_{3}\right)$ can be formulated similarly as (3.9).

\section{The $\bar{\partial}$-Equation and the Frobenius Problem}

In this section, we formulate the $\partial$-equation corresponding to the almost complex structure $J$ which is conjugate to the $\bar{\partial}$-equation. Recall that $J$ is real analytic on $K$, the $\partial$-equation can be complexified into a Frobenius equation. Since $J$ is represented by the $2 \times 2$ matrix $A$, and the entries of $A$ involves the first derivatives of the coordinate functions, the Frobenius equation can be reduced to a first order nonlinear partial differential equation. In the next section we solve the nonlinear equation and prove that $J$ is weakly integrable on the space $K_{0}$ of real analytic knots and in Section 6 we prove that the Frobenius equation is not solvable on $K$. Note that the Frobenius equation is stronger than the $\partial$-equation: When the former is solvable, so is the latter. Conversely, if the $\partial$-equation is solvable and the solutions are real analytic, the complexified solutions satisfy the Frobenius equation. 
4.1. The $\partial$-equation. A few notations are fixed first to formulate the $\partial$ equation. First, since $J$ is an almost complex structure on $K$, for any $\gamma \in K$, $J_{\gamma}^{2}=-I$ as an endormorphism on $T_{\gamma} K$, where $I$ is the identity map. Let $T_{\gamma}^{C} K$ be the complexified tangent space

$$
T_{\gamma}^{C} K=T_{\gamma}^{\prime} K \oplus T_{\gamma}^{\prime \prime} K
$$

where

$$
T_{\gamma}^{\prime} K=\left\{X-i J_{\gamma} X: X \in T_{\gamma} K\right\}
$$

is the $i$-eigenspace of $J_{\gamma}: T_{\gamma}^{C} K \rightarrow T_{\gamma}^{C} K$ and

$$
T_{\gamma}^{\prime \prime} K=\left\{X+i J_{\gamma} X: X \in T_{\gamma} K\right\}
$$

is the $(-i)$-eigenspace of $J_{\gamma}$.

Let $T^{\prime} K=\cup_{\gamma \in K} T_{\gamma}^{\prime} K$. Then $T^{\prime} K$ is a subbundle of $T^{C} K$. It is well-known that $T^{\prime} K$ is closed under the Lie bracket if and only if $J$ is formally integrable, i.e., the Nijenhuis tensor of $J$ vanishes. Similarly $T^{\prime \prime} K=\cup_{\gamma \in K} T^{\prime \prime} K$ is also a subbundle of $T^{C} K$.

For fixed $\gamma \in K^{*}$ and $\tilde{\gamma} \in \mathcal{N}_{\delta}^{1}(\gamma), T_{\gamma}^{\prime} K$ is given as

$$
T_{\tilde{\gamma}}^{\prime} K=\left\{X-i X A: X \in C^{\infty}\left(S^{1}, R^{2}\right)\right\},
$$

in local coordinates, where $A$ is the $2 \times 2$ matrix (3.5). Note that $T_{\bar{\gamma}}^{\prime} K$ is spanned by

$$
\left\{X_{k}-i X_{k} A: k \in N\right\}
$$

since $J^{2}=-I$.

Introduce complex coordinates

$$
\begin{gathered}
z_{k}=x_{k}+i y_{k}, \bar{z}_{k}=x_{k}-i y_{k}, \\
\partial_{z_{k}}=\frac{1}{2}\left(X_{k}-i Y_{k}\right), \\
\partial_{\bar{z}_{k}}=\frac{1}{2}\left(X_{k}+i Y_{k}\right)(k \in N) .
\end{gathered}
$$

$X_{k}-i X_{k} A$ is computed as

$$
(1+b-i a) \partial_{z_{k}}+(1-b-i a) \partial_{\bar{z}_{k}} .
$$

Since $J$ is formally integrable, the collection

$$
\left\{\partial_{z_{k}}+\frac{1-b-i a}{1+b-i a} \partial_{\bar{z}_{k}}: k \in N\right\}
$$


is close under the Lie bracket. Thus elements in (4.6) are commutative. It will be proved in the next section that for any $k \in N$, the $\partial$-equation

$$
\partial_{z_{k}}+\frac{1-b-i a}{1+b-i a} \partial_{\bar{z}_{k}}=\partial_{\zeta_{k}}
$$

is solvable on the space $K_{0}$.

4.2. The Frobenius equation. Recall that $a, b$ are real analytic functions in $\kappa, \tau$, and the coordinate functions $x(\theta), y(\theta)$ and their first derivatives. Without confusion, denote by

$$
a=a(z(\theta), \bar{z}(\theta)), b=b(z(\theta), \bar{z}(\theta)) .
$$

Note that both $a$ and $b$ can be complexified as $a(z(\theta), w(\theta))$ and $b(z(\theta), w(\theta))$, (4.6) can be complexified as

$$
\left\{\partial_{z_{k}}+\frac{1-b(z, w)-i a(z, w)}{1+b(z, w)-i a(z, w)} \partial_{w_{k}}\right\}
$$

on the complexified local patch

$$
\mathcal{N}_{\delta, C}^{1}(\gamma)=\left\{f \in \Gamma\left(N_{\gamma}^{C}\right):\|f\|_{C^{1}}<\delta\right\}
$$

Note that a smooth map on $\mathcal{N}_{\delta, C}^{1}(\gamma)$ can be written as

$$
\phi(z(\theta), w(\theta))=\phi_{0}+\sum_{k=1}^{\infty} \phi_{2 k-1} \sin (2 k \pi \theta)+\phi_{2 k} \cos (2 k \pi \theta)
$$

with $\phi_{k}$ 's are functions in $\left\{z_{k}, w_{k}: k \in N\right\}$. Let $D_{1} \phi=\left(\frac{\partial \phi_{j}}{\partial z_{k}}\right)$ denote the Jacobian matrix. The Frobenius equation is then

$$
\left\{\begin{array}{l}
D_{1} \phi(z, w)=\frac{1-b(z, \phi)-i a(z, \phi)}{1+b(z, \phi)-i a(z, \phi)} \\
\phi(0, w)=w .
\end{array}\right.
$$

The reader may compare our formulation with Lang [12].

4.3. The reduction of the Frobenius equation. To solve the Frobenius equation (4.12), as Lang [12], for any $(z, w) \in \mathcal{N}_{\delta, C}^{1}(\gamma)$, let $\psi$ be the map defined as

$$
\psi(t, z, w)=\phi(t z, w)
$$


By the equation (4.12), $\psi(t, z, w)$ satisfies the following ordinary differential equation in the ét space $C^{\infty}\left(S^{1}, R^{2}\right)$ :

$$
\left\{\begin{array}{l}
\frac{d \psi}{d t}=\frac{(1-b(t z, \psi)-i a(t z, \psi)) z}{1+b(t z, \psi)-i a(t z, \psi)} \\
\psi(0, z, w)=w .
\end{array}\right.
$$

Recall that $a(z(\theta)$ and $w(\theta)), b(z(\theta), w(\theta))$ are $C^{\omega}$-functions in $\kappa, \tau, z(\theta), w(\theta)$ and $z^{\prime}(\theta), w^{\prime}(\theta)$. The Frobenius equation (4.14) involves $\psi, \frac{\partial \psi}{\partial t}$ and $\frac{\partial \psi}{\partial \theta}$, it is a nonlinear partial differential equation of the first order.

Proposition 4. For $z, w \in C^{\infty}\left(S^{1}, R^{2}\right)$, the Frobenius equation (4.12) has a unique solution $\phi(z, w)$ iff (4.14) has a unique solution $\psi(t, z, w)$ for $0 \leq$ $t \leq 1$. The relation between the solutions is

$$
\phi(z, w)=\psi(1, z, w)
$$

Proof. Similar to that of $[\mathbf{1 2}]$ or $[\mathbf{1 1}]$.

\section{The Weak Integrability on $K_{0}$}

In this section, we solve the Frobenius equation (4.12) and prove that $J$ is weakly integrable on the space $K_{0}$ of real analytic knots. By the construction in Section 4, the proof is quite easy by the theorem of Cauchy-Kowalewska. Since $K_{0}$ is equipped with the $C^{\omega}$-topology, we need to pay attention to analytical details. An explanation is also given in the section that the holomorphic functions on $K_{0}$ fail to make a local chart on $K_{0}$ by the inverse theorem of Nash and Moser.

5.1. The $C^{\omega}$-topology on $K_{0}$. The precise definition of $K_{0}$ is given as follows. Let $L_{0}$ be the space of $C^{\omega}$-loops in $R^{3}$ and $L_{0}^{*}$ be the space of imbedded $C^{\omega}$-loops in $R^{3}$. Then the orientation preserving $C^{\omega}$-diffeomorphism group of $S^{1}$ act freely on the space $L_{0}^{*}$ and $K_{0}$ is defined as the quotient space.

The $C^{\omega}$-topology on $L_{0}$ is given as follows. Note that for any $\gamma(t) \in L_{0}$, $\gamma(t)$ can be extended analytically over a certain annulus

$$
A_{\epsilon_{0}}=\left\{z \in C: 1-\epsilon_{0}<|z|<1+\epsilon_{0}\right\} \text {. }
$$

Let

$$
\mathcal{N}_{\epsilon, \delta}(\gamma)=\left\{\tilde{\gamma} \in L_{0}:\|\tilde{\gamma}-\gamma\|_{C^{0}\left(A_{\epsilon}\right)}<\delta\right\}
$$

with $\epsilon<\epsilon_{0}$. As Brylinski [5], the collection

$$
\left\{\mathcal{N}_{\epsilon, \delta}(\gamma): \epsilon>0, \delta>0\right\}
$$


define a local basis of the $C^{\omega}$-topology at $\gamma$. Note that $\gamma \in L$ is in $L_{0}$ iff the arc-length parametrization $\gamma(s)$ or the standard parametrization $\gamma(\theta)$ is $C^{\omega}$.

As Lemma 2.1 , let $L_{0}^{\prime}$ be the space of $C^{\omega}$-loops with curvature $\kappa>0$ everywhere. Then $L_{0}^{\prime}$ is an open and dense set in $L_{0}$. Thus the collection

$$
\left\{\mathcal{N}_{\epsilon, \delta}(\gamma): \gamma \in L_{0}^{\prime}, \epsilon>0, \delta>0\right\}
$$

gives an open cover on $L_{0}$ and hence a local coordinate system on $L_{0}$. Note that the $C^{\omega}$-topology is a finer one than the smooth Fréchet topology, because by the Cauchy formula, for any $\epsilon, \epsilon^{\prime}$ with $0<\epsilon<\epsilon^{\prime}$, all the $C^{n}$-norm of $\gamma \in L_{0}$ on $A_{\epsilon}$ can be bounded by $\|\gamma\|_{C^{0}\left(A_{\epsilon^{\prime}}\right)}$, as Theorem 14.6 of [20].

Descending to the quotient topology, for any $\gamma \in K_{0}$, define again $\mathcal{N}_{\epsilon, \delta}(\gamma)$ by (5.1). Then the collection

$$
\left\{\mathcal{N}_{\epsilon, \delta}(\gamma): \gamma \in K_{0}^{\prime}, \epsilon>0, \delta>0\right\}
$$

is an open cover on $K_{0}$ and gives a local coordinate system on $K_{0}$. As (2.5), (2.8) and (2.9), let $x(\theta)$ and $y(\theta)$ denote the local coordinate functions, and

$$
\left\{X_{k}, Y_{k}: k \in N\right\}
$$

be the local basis on $\mathcal{N}_{\epsilon, \delta}(\gamma)$.

5.2. The weak integrability on $K_{0}$. For any $\gamma \in K_{0}$, the tangent space $T_{\gamma} K_{0}$ is the space $\Gamma_{0}\left(N_{\gamma}\right)$ of $C^{\omega}$-sections of the normal bundle $N_{\gamma}$. Let $J$ be defined as the rotation in $\frac{\pi}{2}$ in $\Gamma_{0}\left(N_{\gamma}\right)$. The computations in Section 4 can be translated on $K_{0}$; As (3.7), $J$ is represented by the $2 \times 2$ matex $A$ with entries (3.4).

Proposition 5.1. $J$ is a well-defined, formally integrable almost complex structure on $K_{0}$ and is real analytic.

Proof. For any $\epsilon, \epsilon^{\prime}$ with $0<\epsilon<\epsilon^{\prime}$, since

$$
\left\|z^{(k)}\right\|_{C^{0}\left(A_{\epsilon}\right)} \leq C\|z\|_{C^{0}\left(A_{\epsilon^{\prime}}\right)},
$$

for any $k \in N$, the matrix $A$ defines a smooth map in the $C^{\omega}$-topology. Thus $J$ is well-defined on $K_{0} . J$ is formally integrable by Brylinski $[\mathbf{3}, 4]$. With $J$ given explicitly as (3.4), the proof of the analyticity is omitted.

Theorem 5.2 (Drinfeld, LeBrun). The almost complex structure $J$ is weakly integrable on the space $K_{0}$, i.e., for any $k \in N$, the $\partial$-equation (4.7) is solvable and the holomorphic differentials $\left\{\partial_{\zeta_{k}}: k \in N\right\}$ is weakly dense in $T^{*} K_{0}$.

Proof. By Proposition 5.1, $J$ is real analytic on $K_{0}$. As Section 4, the $\partial$ equation can be complexified into a Frobenius equation and the Frobenius 
equation can be further reduced to a first order nonlinear partial differential equation

$$
\left\{\begin{array}{l}
\frac{\partial \psi}{\partial t}=\frac{1-b(t z, \psi)-i a(t z, \psi)}{1+b(t z, \psi)-i a(t z, \psi)} z \\
\psi(0, z, w)=w
\end{array}\right.
$$

Note that, for any $\gamma \in K_{0}^{*}, \kappa, \tau$ are both real analytic functions in $\theta$, and for any $(z, w) \in C^{\omega}\left(S^{1}, R^{2}\right)$, the nonlinear $\operatorname{PDE}(5.6)$ is a real analytic system. By the theorem of Cauchy-Kowalewska, (5.6) has a unique solution $\psi(t, z, w)$ for small $t \geq 0$. By rescaling, $\psi(t, z, w)$ is defined on $0 \leq t \leq 1$. By Proposition 4, the Frobenius equation

$$
\left\{\begin{array}{l}
D_{1} \phi=\frac{1-b(z, \phi)-i a(z, \phi)}{1+b(z, \phi)-i a(z, \phi)} \\
\phi(0, w)=w
\end{array}\right.
$$

has a unique solution for any $(z, w) \in \mathcal{N}_{\epsilon, \delta}(\gamma) \otimes C$ when $\delta>0$ is small. The $\partial$-equation is thus solvable, the holomorphic functions are given by $\zeta_{k}=z_{k}+\phi_{k}(z, \bar{z})$.

Let $\Phi$ be the map on $\mathcal{N}_{\epsilon, \delta}(\gamma)$ defined as

$$
\Phi\left(z_{k}\right)=z_{k}+\phi_{k}(z, \bar{z}) .
$$

Notice that

$$
D \Phi(z, \bar{z})=\frac{2(1-i a)}{1+b-i a}
$$

involves the first derivatives of the coordinate functions. $D \Phi$ is invertible on $\mathcal{N}_{\epsilon, \delta}(\gamma)$. Thus the germ of holomorphic differentials is weakly dense in $T^{*} K_{0}$. Theorem 5.2 is thus proved.

5.3. On the inverse function theorem. In this section, it is shown that the inverse function theorem of Nash and Moser fails to implies that $\Phi$ defined as (5.8) is a local diffeomorphism and thus $J$ is integrable on $K$. The reader may refer to Hamilton [8] for the exact statement of the inverse function theorem. Roughly speaking, the inverse function theorem works in the tame category. As in Hamilton [8], the space $C^{\omega}\left(S^{1}, R^{2}\right)$ with the $C^{\omega}$-topology is a tame Fréchet space. It will be shown that the map $\Phi$ fails to satisfy the tameness conditions.

It is a remark that however $\Phi$ and the inverse of $D \Phi$ both satisfy the tameness estimates in the $C^{\infty}$-topology. By (5.9), the inverse of $D \Phi$ is an ordinary differential operator. As Corollary 2.2.7 of Part II of [8], the inverse 
is a tame map on $\mathcal{N}_{\epsilon, \delta}(\gamma)$. To prove that $\Phi$ is tame, we solve the equation (5.6). (We will return to this practise of solving (5.6) more specifically in Section 6, and here we are brief.) As in Garabedian [6] and John [9], (5.6) can be solved by integrating a system of ordinary differential equations which describes the characteristic curves with initial conditions given by $w$. As Theorem 3.2.1 of Part II of [8], the tameness estimates of $\Phi$ can be easily established.

Proposition 5.3. The inverse of $D \Phi$ defined as (5.9) is not a tame map in the $C^{\omega}$-topology.

Proof. Consider the Frobenius equation around the circle

$$
\gamma(\theta)=\frac{1}{2 \pi}(\cos (2 \pi \theta), \sin (2 \pi \theta), 0)
$$

and let $x(\theta)=0$. Note that $l=1, \kappa=2 \pi, \tau=0$,

$$
\lambda_{1}=1, \lambda_{2}=0, \lambda_{3}=y^{\prime}(\theta),
$$

the matrix $A$ has the the following explicit entries:

$$
\begin{gathered}
a=0, b=\sqrt{1+y^{\prime}(\theta)^{2}}, \\
c=-\frac{1}{\sqrt{1+y^{\prime}(\theta)^{2}}}, d=0 .
\end{gathered}
$$

Thus the inverse of $D \Phi$ is computed as

$$
(D \Phi)_{\mid(0, y)}^{-1}=\frac{1}{2}\left(1+\sqrt{1+{y^{\prime}}^{2}}\right) \text {. }
$$

Note that $(D \Phi)^{-1}$ has a nonlinear term $y^{\prime 2}$. As Example 2.1.3 of Part II of $[8]$, it is not a tame map in the $C^{\omega}$-topology.

\section{The Frobenius Problem on $\mathrm{K}$}

In this section, we give an explicit form of the Frobenius equation

$$
\left\{\begin{array}{l}
\frac{\partial \psi}{\partial t}=\frac{1-b(t z, \psi)-i a(t z, \psi)}{1+b(t z, \psi)-i a(t z, \psi)} z \\
\psi(0, z, w)=w
\end{array}\right.
$$

and prove an insolvability of the equation. By Proposition 4, the Frobenius problem on $K$ is thus not integrable. 
6.1. An explicit form of the Frobenuis equation. Consider the Frobenius equation (6.1) around the standard circle

$$
\gamma(\theta)=(\cos (2 \pi \theta), \sin (2 \pi \theta), 0) .
$$

Then $l=2 \pi, \kappa=1, \tau=0$,

$$
\lambda_{1}=2 \pi(1-x(\theta)), \lambda_{2}=x^{\prime}(\theta), \lambda_{3}=y^{\prime}(\theta) .
$$

Introducing

$$
\mu_{2}=\frac{\lambda_{2}}{\lambda_{1}}, \mu_{3}=\frac{\lambda_{3}}{\lambda_{1}}
$$

the matrix $A$ has the following entries:

$$
\begin{gathered}
a(z, \bar{z})=\frac{\mu_{2} \mu_{3}}{\sqrt{1+\mu_{2}^{2}+\mu_{3}^{2}}}, \\
b(z, \bar{z})=\frac{1+\mu_{3}^{2}}{\sqrt{1+\mu_{2}^{2}+\mu_{3}^{2}}}, \\
c(z, \bar{z})=-\frac{1+\mu_{2}^{2}}{\sqrt{1+\mu_{2}^{2}+\mu_{3}^{2}}}, \\
d(z, \bar{z})=-\frac{\mu_{2} \mu_{3}}{\sqrt{1+\mu_{2}^{2}+\mu_{3}^{2}}} .
\end{gathered}
$$

All the entries can be complexified as $a=a(z, w)$ etc..

To find a simple form of the equation, let $z=\delta>0$. Then

$$
\begin{gathered}
1+\mu_{2}^{2}+\mu_{3}^{2}=1+\frac{z^{\prime} w^{\prime}}{\lambda^{2}}=1, \\
1+b-i a=2+\mu_{3}^{2}-i \mu_{2} \mu_{3}=2, \\
1-b-i a=-\mu_{3}^{2}-i \mu_{2} \mu_{3}=\frac{w^{\prime 2}}{2 \lambda_{1}^{2}} .
\end{gathered}
$$

The equation (6.1) has the explicit form

$$
\left\{\begin{array}{l}
\frac{\partial \psi}{\partial t}=\frac{\delta\left(\frac{\partial \psi}{\partial \theta}\right)^{2}}{16 \pi^{2}(1-\delta t-\psi)^{2}} \\
\psi_{\mid t=0}=w(\theta) .
\end{array}\right.
$$

Introduce $\varphi=\frac{1}{1-\delta t-\psi}$ and $v=\frac{1}{1-w}$. Denote again by $t$ for $\frac{t}{16 \pi^{2}}$. Then (6.6) is converted as

$$
\left\{\begin{array}{l}
\frac{\partial \varphi}{\partial t}=\varphi^{2}+\left(\frac{\partial \varphi}{\partial \theta}\right)^{2} \\
\psi_{\mid t=0}=v
\end{array}\right.
$$


6.2. A simpler example. The equation (6.7) is a first order, nonlinear equation. As in Garabedian [6] and John [9], when $w$ is a real function, (6.6) can be explicitly solved by integrating a system of ordinary differential equations which describes the characteristic curves. This is also the case when $w$ is real analytic. To show that the Frobenius equation is not solvable, (6.7) will be shown unsolvable for certain $v$. To illustrate the idea of proof, consider first the equation

$$
\left\{\begin{array}{l}
\frac{\partial \psi}{\partial t}=\left(\frac{\partial \psi}{\partial \theta}\right)^{2} \\
\psi_{\mid t=0}=v
\end{array}\right.
$$

Proposition 6.1. Let $v(\theta)$ be a smooth function on $S^{1}$ with $\operatorname{Im} v^{\prime}(\theta) \neq 0$ on $S^{1}$. Then the equation (6.8) is solvable iff $v \in C^{\omega}$.

Proof. The "if" part is by the theorem of Cauchy-Kowalevska. To prove the "only if" part, note that (6.8) can be explicitly solved. Let $\eta=\frac{\partial \psi}{\partial \theta}$. Then (6.8) is converted into the quasi-linear Burger equation

$$
\left\{\begin{array}{l}
\frac{\partial \eta}{\partial t}=2 \eta \frac{\partial \eta}{\partial \theta} \\
\eta_{\mid t=0}=v^{\prime}
\end{array}\right.
$$

and the solution is given as

$$
\eta(t, \theta)=v^{\prime}(\theta+2 t \eta(t, \theta)) .
$$

Assume that $v^{\prime}$ is never real and (6.8) has a solution $\eta(\theta, t)$. Then for small $t, \eta(\theta, t)$ is also never real. By $(6.10), v^{\prime}$ is extended over a certain annulus $A_{\epsilon}$. To show that the extension is holomorphic, let

$$
\eta=\eta_{1}+i \eta_{2}, v^{\prime}=v_{1}^{\prime}+i v_{2}^{\prime}
$$

and rewrite $(6.10)$ as

$$
\eta(t, \theta)=v^{\prime}\left(\theta+2 t \eta_{1}, 2 t \eta_{2}\right) .
$$

Differentiating the explicit function (6.12),

$$
\left\{\begin{array}{l}
\frac{\partial \eta}{\partial t}_{\mid t=0}=2 \eta_{1} \frac{\partial v}{\partial \zeta_{1}}+2 \eta_{2} \frac{\partial v}{\partial \zeta_{2}} \\
\frac{\partial \eta}{\partial \theta}_{\mid t=0}=\frac{\partial v}{\partial \zeta_{1}} .
\end{array}\right.
$$

Substituting (6.13) to $(6.9)$,

$$
\eta_{2}\left(\frac{\partial v^{\prime}}{\partial \zeta_{1}}+i \frac{\partial v^{\prime}}{\partial \zeta_{2}}\right)=0
$$

Since $\eta_{2} \neq 0$ for small $t, v^{\prime}$ satisfies the Cauchy-Riemann equation, $v^{\prime} \in C^{\omega}$. 
6.3. An unsolvability of the Frobenius equation. Denote by $S$ the set of complex valued, smooth functions $v(\theta)$ on $S^{1}$ such that

$$
v^{\prime} \neq 0, \operatorname{Im} \frac{v^{2}+v^{\prime 2}}{v^{\prime}} \neq 0
$$

on $S^{1}$. The following proposition shows that the Frobenius equation is not solvable on the knot space $K$.

Proposition 6.2. (6.7) is unsolvable for generic $v \in S$.

Proof. To find the general solution for (6.7), let $p=\frac{\partial \varphi}{\partial \theta}, q=\frac{\partial \varphi}{\partial t}$ and rewrite the equation as

$$
F(\theta, t, \varphi, p, q)=q-\varphi^{2}-p^{2}=0
$$

As [6] and [9], (6.7) is solved by integrating

$$
\left\{\begin{array}{l}
\frac{d \theta}{d s}=F_{p}=-2 p \\
\frac{d t}{d s}=F_{q}=1 \\
\frac{d \varphi}{d s}=p F_{p}+q F_{q}=q-2 p^{2} \\
\frac{d p}{d s}=-F_{\theta}-p F_{\varphi}=2 p \varphi \\
\frac{d q}{d s}=-F_{t}-q F_{\varphi}=2 q \varphi
\end{array}\right.
$$

with the initial condition

$$
\left\{\begin{array}{l}
\theta_{\mid s=0}=\tau \\
t_{\mid s=0}=0 \\
\varphi_{\mid s=0}=v(\tau) \\
p_{\mid s=0}=v^{\prime}(\tau) \\
q_{\mid s=0}=v^{2}(\tau)+v^{\prime 2}(\tau) .
\end{array}\right.
$$

Where $s$ and $\tau$ are two parameters.

To integrate (6.16), note first that (6.16) implies $t=s$. The last two equations of (6.16) imply that $\underset{q}{p}$ is independent of $s$,

$$
q=\frac{v^{2}+v^{\prime 2}}{v^{\prime}} p
$$


Substituting (6.18) to $(6.16),(6.16)$ is reduced as

$$
\left\{\begin{array}{l}
\frac{d \theta}{d t}=-2 p \\
\frac{d \varphi}{d t}=\frac{v^{2}+v^{\prime 2}}{v^{\prime}} p-2 p^{2} \\
\frac{d p}{d t}=2 p \varphi
\end{array}\right.
$$

Let $\lambda$ and $\mu$ be functions defined as

$$
\lambda(\tau)=\frac{v^{2}+v^{\prime 2}}{v^{\prime}}, \mu(\tau)=\frac{v}{v^{\prime}} .
$$

Then the equation (6.19) implies

$$
\frac{d \varphi}{d p}=\frac{\lambda-2 p}{2 \psi}, \varphi^{2}=\lambda p-p^{2} .
$$

Substituting (6.21) to (6.19), $p$ is integrated as

$$
\begin{gathered}
\frac{d p}{d t}=2 p \sqrt{\lambda p-p^{2}} \\
t=\int_{v^{\prime}}^{p} \frac{d u}{2 u \sqrt{\lambda u-u^{2}}},
\end{gathered}
$$

$$
p(\tau, t)=\frac{\lambda(\tau)}{1+(\lambda t-\mu)^{2}} .
$$

Substituting (6.21) to (6.19), (6.7) is solved;

$$
\left\{\begin{array}{l}
\theta(\tau, t)=\tau-\left.2 \tan ^{-1}(\lambda u-\mu)\right|_{0} ^{t} \\
\varphi(\tau, t)=-\frac{\lambda(\lambda t-\mu)}{1+(\lambda t-\mu)^{2}} .
\end{array}\right.
$$

Note that $\tau=\tau(\theta, t)$ is determined by (6.23A) implicitly.

When $\operatorname{Im} \lambda \neq 0$ on $S^{1}$, similar to the case of Burger equation, if the equation (6.7) has a solution, then (6.23A) implies that $\tau$ is a complex variable and thus $\mu(\tau), \lambda(\tau)$ are both forced to extend over a certain annulus $A_{\epsilon}$. Assume that $\lambda$ and $\mu$ are extended as

$$
\lambda=\lambda(\tau, \bar{\tau}), \mu=\mu(\tau, \bar{\tau}) .
$$


Let $z(\tau, t)$ and $\gamma(\tau, t)$ be functions defined as

$$
z(\tau, t)=\lambda t-\mu, \gamma(\tau, t)=\frac{2 \lambda}{1+z^{2}} .
$$

Introduce

$$
\alpha=1-\frac{2}{1+z^{2}} \frac{\partial z}{\partial \tau}-\frac{2}{1+\mu^{2}} \frac{\partial \mu}{\partial \tau}
$$

$$
\beta=-\frac{2}{1+z^{2}} \frac{\partial z}{\partial \bar{\tau}}-\frac{2}{1+\mu^{2}} \frac{\partial \mu}{\partial \bar{\tau}} .
$$

Differentiating (6.23A),

$$
\begin{aligned}
& \frac{\partial \tau}{\partial t}=\frac{\bar{\alpha} \gamma-\beta \bar{\gamma}}{|\alpha|^{2}-|\beta|^{2}} \\
& \frac{\partial \tau}{\partial \theta}=\frac{\bar{\alpha}-\beta}{|\alpha|^{2}-|\beta|^{2}}
\end{aligned}
$$

Note that $\alpha \sim 1$ and $\beta \sim 0$ for small $t$.

Differentiating (6.23B) follows that

$$
\frac{\partial \varphi}{\partial t}=\frac{-z}{1+z^{2}} \frac{\partial \lambda}{\partial t}-\frac{\lambda\left(1-z^{2}\right)}{\left(1+z^{2}\right)^{2}} \frac{\partial z}{\partial t} .
$$

Substitute

$$
\begin{aligned}
& \frac{\partial z}{\partial t}=t \frac{\partial \lambda}{\partial t}-\frac{\partial \mu}{\partial t}+\lambda \\
& \frac{\partial \lambda}{\partial t}=\frac{\partial \lambda}{\partial \tau} \frac{\partial \tau}{\partial t}+\frac{\partial \lambda}{\partial \bar{\tau}} \frac{\partial \bar{\tau}}{\partial t} \\
& \frac{\partial \mu}{\partial t}=\frac{\partial \mu}{\partial \tau} \frac{\partial \tau}{\partial t}+\frac{\partial \mu}{\partial \bar{\tau}} \frac{\partial \bar{\tau}}{\partial t}
\end{aligned}
$$

and (6.27) to (6.28). $\frac{\partial \varphi}{\partial t}-\varphi^{2}$ is then computed as

$$
\begin{gathered}
\frac{-\lambda^{2}}{\left(1+z^{2}\right)^{2}}-\frac{\bar{\alpha} \gamma-\beta \bar{\gamma}}{|\alpha|^{2}-|\beta|^{2}}\left\{\frac{-\mu z^{2}+2 z+\mu}{\left(1+z^{2}\right)^{2}} \frac{\partial \lambda}{\partial \tau}-\frac{\lambda\left(1-z^{2}\right)}{\left(1+z^{2}\right)^{2}} \frac{\partial \mu}{\partial \tau}\right\} \\
-\frac{\alpha \bar{\gamma}-\bar{\beta} \gamma}{|\alpha|^{2}-|\beta|^{2}}\left\{\frac{-\mu z^{2}+2 z+\mu}{\left(1+z^{2}\right)^{2}} \frac{\partial \lambda}{\partial \bar{\tau}}-\frac{\lambda\left(1-z^{2}\right)}{\left(1+z^{2}\right)^{2}} \frac{\partial \mu}{\partial \bar{\tau}}\right\}
\end{gathered}
$$


Similarly $\frac{\partial \varphi}{\partial \theta}$ is computed as

$$
\begin{gathered}
-\frac{\bar{\alpha}-\beta}{|\alpha|^{2}-|\beta|^{2}}\left\{\frac{-\mu z^{2}+2 z+\mu}{\left(1+z^{2}\right)^{2}} \frac{\partial \lambda}{\partial \tau}-\frac{\lambda\left(1-z^{2}\right)}{\left(1+z^{2}\right)^{2}} \frac{\partial \mu}{\partial \tau}\right\} \\
-\frac{\alpha-\bar{\beta}}{|\alpha|^{2}-|\beta|^{2}}\left\{\frac{-\mu z^{2}+2 z+\mu}{\left(1+z^{2}\right)^{2}} \frac{\partial \lambda}{\partial \bar{\tau}}-\frac{\lambda\left(1-z^{2}\right)}{\left(1+z^{2}\right)^{2}} \frac{\partial \mu}{\partial \bar{\tau}}\right\} .
\end{gathered}
$$

Note that $\beta$ is a linear function in $\frac{\partial \lambda}{\partial \bar{\tau}}$ and $\frac{\partial \mu}{\partial \bar{\tau}}, \alpha$ is a similar function in $\frac{\partial \lambda}{\partial \tau}$ and $\frac{\partial \mu}{\partial \tau}$. Multiplying the equation (6.7) by

$$
\left(|\alpha|^{2}-|\beta|^{2}\right)^{2}\left(1+z^{2}\right)^{4}\left(1+\bar{z}^{2}\right)^{2}
$$

it follows that $\frac{\partial \lambda}{\partial \bar{\tau}}, \frac{\partial \mu}{\partial \bar{\tau}}$ and $t$ satisfy a polynomial equation

$$
P\left(t, \lambda, \mu, \frac{\partial \lambda}{\partial \tau}, \frac{\partial \mu}{\partial \tau}, \frac{\partial \lambda}{\partial \bar{\tau}}, \frac{\partial \mu}{\partial \bar{\tau}}\right)=0
$$

Consider $P$ as a polynomial in $\frac{\partial \lambda}{\partial \bar{\tau}}$ and $\frac{\partial \mu}{\partial \bar{\tau}}$. Note that, when

$$
\frac{\partial \lambda}{\partial \bar{\tau}}=0, \frac{\partial \mu}{\partial \bar{\tau}}=0
$$

$\lambda, \mu$ and thus $v$ and $v^{\prime}$ are holomorphically extended, (6.23) gives a solution to $(6.7)$. Hence $(6.34)$ is a solution to $(6.33)$, the constant part $P_{0}$ of $P$ vanishes identically.

$$
P_{0}\left(t, \lambda, \mu, \frac{\partial \lambda}{\partial \tau}, \frac{\partial \mu}{\partial \tau}\right)=0
$$

Let $H=P-P_{0}$.

By (6.31) and (6.32), $P_{0}$ can be easily computed as a complete square. Valued at $t=0,(6.35)$ implies the equation

$$
\mu \frac{\partial \lambda}{\partial \tau}+\frac{\lambda\left(1-\mu^{2}\right)}{1+\mu^{2}} \frac{\partial \mu}{\partial \tau}=\lambda
$$

(In fact, (6.35) and (6.36) are equivalent.) Consider the equation $H=0$. Valued at $t=0$, the equation implies either the linear equation

$$
\mu \frac{\partial \lambda}{\partial \bar{\tau}}+\frac{\lambda\left(1-\mu^{2}\right)}{1+\mu^{2}} \frac{\partial \mu}{\partial \bar{\tau}}=0
$$

or the linear equation

$$
\mu \frac{\partial \lambda}{\partial \bar{\tau}}+\frac{\lambda\left(1-\mu^{2}\right)}{1+\mu^{2}} \frac{\partial \mu}{\partial \bar{\tau}}=\frac{2 \bar{\lambda}}{1+\bar{\mu}^{2}} .
$$


The vanishing of the coefficient of the highest $t$-power in $H$ implies the equation

$$
\begin{gathered}
\left(1-\frac{2}{1+\mu^{2}} \frac{\partial \mu}{\partial \tau}+\frac{2}{1+\bar{\mu}^{2}} \frac{\overline{\partial \mu}}{\partial \bar{\tau}}\right)\left(-\mu \frac{\partial \lambda}{\partial \bar{\tau}}+\lambda \frac{\partial \mu}{\partial \bar{\tau}}\right) \\
+\frac{2}{1+\mu^{2}} \frac{\partial \mu}{\partial \bar{\tau}}\left(-\mu \frac{\partial \lambda}{\partial \tau}+\lambda \frac{\partial \mu}{\partial \tau}\right)=0 .
\end{gathered}
$$

Where the first and second term of (6.38) are derived from those terms of (6.32), since the highest $t$-power appears in the square of (6.32). By (6.36) and (6.37A), substituting $\frac{\partial \lambda}{\partial \tau}$ and $\frac{\partial \lambda}{\partial \bar{\tau}}$ to $(6.38)$,

$$
\frac{2}{1+\bar{\mu}^{2}} \frac{2 \lambda}{1+\mu^{2}}\left|\frac{\partial \mu}{\partial \bar{\tau}}\right|^{2}=0
$$

Thus $\frac{\partial \mu}{\partial \bar{\tau}}=0, \frac{\partial \lambda}{\partial \bar{\tau}}=0 . v \in C^{\omega}$.

The complicated case is the equation $(6.37 \mathrm{~B})$. In this case, substituting again $(6.36)$ and $(6.37 \mathrm{~B})$ to $(6.38)$ follows that $\frac{\partial \mu}{\partial \tau}$ is a quadratic equation in $\frac{\partial \mu}{\partial \bar{\tau}}$.

$$
\frac{\partial \mu}{\partial \tau}=Q\left(\lambda, \mu, \frac{\partial \mu}{\partial \bar{\tau}}\right)
$$

To complete the proof of Proposition 6.2, it will be shown that, the equation $H=0$ implies two more nontrivial equations which can be reduced to different polynomial equations

$$
\begin{aligned}
& \Gamma_{1}\left(\lambda, \mu, \frac{\partial \mu}{\partial \bar{\tau}}\right)=0 \\
& \Gamma_{2}\left(\lambda, \mu, \frac{\partial \mu}{\partial \bar{\tau}}\right)=0
\end{aligned}
$$

which are in the single variable $\frac{\partial \mu}{\partial \bar{\tau}}$ by $(6.36),(6.37 \mathrm{~B})$ and $(6.40)$. Thus by restricting $\lambda$ and $\mu$ on the real line $\tau=\bar{\tau}$, for generic $v(\theta),(6.41)$ has no solution. (The resultant can be used to check that $\Gamma_{1}$ and $\Gamma_{2}$ have no common solutions.)

The two polynomials can be indeed chosen in the following way. Regard $H$ as a polynomial in $z$ instead of $t$. Then the two polynomials are deduced from the constant term and the coefficient of $z$. An explicit calculation can be given to show that the polynomials are in fact different; the calculation is lengthy however not difficult, may we omit the details here. Proposition 6.2 is thus proved. 
Corollary 6.3. The Frobenius problem on $K$ is not integrable.

\section{References}

[1] M.F. Atiyah, The geometry and physics of knots, Lezioni Lincee, Cambridge University Press, 1990.

[2] R. Bott, The space of loops on a Lie group, Michigan Math. J., 5 (1958).

[3] J.-L. Brylinski, The Kähler geometry of knots in a smooth threefold, Preprint of Penn. State Univ., No. PM93 (1990).

[4] J.-L. Brylinski, The line bundle on the space of singular knots, Preprint of Penn. State Univ., 1991.

[5] J.-L. Brylinski, Loop spaces, characteristic classes and geometric quantization, Birkhauser Boston 1993.

[6] P.R. Garabedian, Partial differential equations, John Wiley and Sons Inc. 1964.

[7] A. Grothendieck, Espaces vectoriels topologiques, Publicacao da Matematica de S. Paulo, 1964.

[8] R. Hamilton, The inverse function theorem of Nash and Moser, Bull. AMS 7 (New Series), No. 1.

[9] F. John, Partial differential equations, Fourth Ed., Springer-Verlag 1982.

[10] A.A. Krillov, Kähler structure on the orbits of the group of diffeomorphisms of the circle, Funk. Anal. Prilozheniya, 21 (1987), 42-45.

[11] S. Kobayashi and K. Nomizu, Foundations of differential geometry, Vol. II, John Wiley and Sons, 1969.

[12] S Lang, Differential manifolds, Addison Wesley Publishing Company, Second Ed., 1985.

[13] C. LeBrun, Spaces of null geodesics in complex-Riemannian geometry, Trans. Amer. Soc., 278 (1983), 209-231.

[14] C. LeBrun, A Kähler structure on the space of world-sheets, Preprint, 1993.

[15] L. Lempert, Loop spaces as complex manifolds, Preprint, 1992.

[16] J. Milnor, Remarks on infinite dimensional Lie groups, In Relativity, groups and topology, Houches Session XL 1983, North-Holland, Amsterdan, 1984.

[17] A. Newlander and L. Nirenberg, Complex analytic coordinates in almost complex manifolds, Ann. Math., 65 (1957), 391-404.

[18] J.-P. Penot, Sur le théoreme de Frobenius, Bull. Soc. Math. France, 98 (1970), 47-80.

[19] A. Pressley and G. Segal, Loop groups, Oxford University Press 1986.

[20] W. Rudin, Real and complex analysis, McGraw-Hill Inc. 1966.

[21] R.G. Wang, Essays on vortices, knots and monopoles, Thesis, Harvard University 1993.

Received March 23, 1993 and revised February 3, 1994.

Hong Kong Univ. of Science and Technology

Clear Water Bay

KOWLOON, HONG Kong 



\title{
PACIFIC JOURNAL OF MATHEMATICS
}

Founded by E. F. Beckenbach (1906-1982) and F. Wolf (1904-1989)

\section{EDITORS}

\author{
Sun-Yung Alice Chang (Managing Editor) \\ University of California \\ Los Angeles, CA 90095-1555 \\ pacific@math.ucla.edu
}

\section{F. Michael Christ}

University of California

Los Angeles, CA 90095-1555

christ@math.ucla.edu

Thomas Enright

University of California

San Diego, La Jolla, CA 92093

tenright@ucsd.edu

Nicholas Ercolani

University of Arizona

Tucson, AZ 85721

ercolani@math.arizona.edu
Robert Finn

Stanford University

Stanford, CA 94305

finn@gauss.stanford.edu

Vaughan F. R. Jones

University of California

Berkeley, CA 94720

vfr@math.berkeley.edu

Steven Kerckhoff

Stanford University

Stanford, CA 94305

spk@gauss.stanford.edu
Martin Scharlemann

University of California

Santa Barbara, CA 93106

mgscharl@math.ucsb.edu

Gang Tian

Courant Institute

New York University

New York, NY 10012-1100

tiang@taotao.cims.nyu.edu

V. S. Varadarajan

University of California

Los Angeles, CA 90095-1555

vsv@math.ucla.edu

\section{SUPPORTING INSTITUTIONS}

\section{CALIFORNIA INSTITUTE OF TECHNOLOGY \\ NEW MEXICO STATE UNIVERSITY \\ OREGON STATE UNIVERSITY \\ STANFORD UNIVERSITY \\ UNIVERSITY OF ARIZONA \\ UNIVERSITY OF BRITISH COLUMBIA \\ UNIVERSITY OF CALIFORNIA UNIVERSITY OF HAWAII}

\author{
UNIVERSITY OF MONTANA \\ UNIVERSITY OF NEVADA, RENO \\ UNIVERSITY OF OREGON \\ UNIVERSITY OF SOUTHERN CALIFORNIA \\ UNIVERSITY OF UTAH \\ UNIVERSITY OF WASHINGTON \\ WASHINGTON STATE UNIVERSITY
}

The supporting Institutions listed above contribute to the cost of publication of this Journal, but they are not owners or publishers and have no responsibility for its contents or policies.

Manuscripts must be prepared in accordance with the instructions provided on the inside back cover.

The Pacific Journal of Mathematics (ISSN 0030-8730) is published monthly except for July and August. Regular subscription rate: $\$ 215.00$ a year (10 issues). Special rate: $\$ 108.00$ a year to individual members of supporting institutions.

Subscriptions, orders for back issues published within the last three years, and changes of subscribers address should be sent to Pacific Journal of Mathematics, P.O. Box 4163, Berkeley, CA 94704-0163, U.S.A. Prior back issues are obtainable from Kraus Periodicals Co., Route 100, Millwood, NY 10546.

The Pacific Journal of Mathematics at the University of California, c/o Department of Mathematics, 981 Evans Hall, Berkeley, CA 94720 (ISSN 0030-8730) is published monthly except for July and August. Second-class postage paid at Berkeley, CA 94704, and additional mailing offices. POSTMASTER: send address changes to Pacific Journal of Mathematics, P.O. Box 6143, Berkeley, CA 94704-0163.

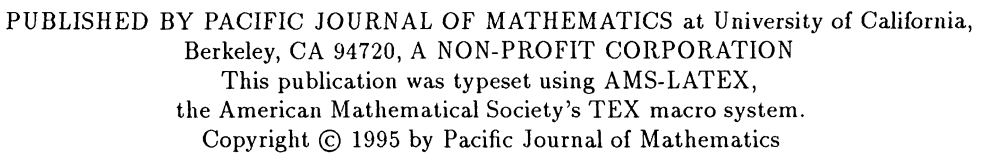




\section{PACIFIC JOURNAL OF MATHEMATICS}

\section{Volume $171 \quad$ No. $2 \quad$ December 1995}

On $H^{p}$-solutions of the Bezout equation

ERIC AMAR, JOAQUIM BRUNA FLORIS and ARTUR NiCOLAU

Amenable correspondences and approximation properties for von Neumann algebras

309

Claire ANANTHARAman-DElaroche

On moduli of instanton bundles on $\mathbb{P}^{2 n+1}$

VinCENZO ANCONA and Giorgio MaRia OtTAVIANI

Minimal surfaces with catenoid ends

JORGEN BERGLUND and WAYNE ROSSMAN

Permutation model for semi-circular systems and quantum random walks

PHILIPPE BIANE

The Neumann problem on Lipschitz domains in Hardy spaces of order less than one

RUSSELL M. BROWN

Matching theorems for twisted orbital integrals

409

REBECCA A. HERB

Uniform algebras generated by holomorphic and pluriharmonic functions on strictly

pseudoconvex domains

ALEXANDER IzZO

Quantum Weyl algebras and deformations of $U(g)$

NAIHUAN JING and JAMES ZHANG

Calcul du nombre de classes des corps de nombres

STÉPHANE LOUBOUTIN

On geometric properties of harmonic Lip ${ }_{1}$-capacity

PertTi MatTila and P. V. PARAmonov

Reproducing kernels and composition series for spaces of vector-valued holomorphic functions

Bent ØRsted and Genkai Zhang

Iterated loop modules and a filtration for vertex representation of toroidal Lie algebras

S. ESWARA RAO

The intrinsic mountain pass

MARTIN SCHECHTER

A Frobenius problem on the knot space

RON G. WANG

On complete metrics of nonnegative curvature on 2-plane bundles

DAVID YANG

Correction to: "Free Banach-Lie algebras, couniversal Banach-Lie groups, and more"

VLADIMIR G. PESTOV

Correction to: "Asymptotic radial symmetry for solutions of $\Delta u+e^{u}=0$ in a punctured disc"

KAI Seng (KAISing) CHOU (Tso) and Tom YAU-Heng WAN 